\title{
Elevated Expression of PDZD11 Is Associated With Poor Prognosis and Immune Infiltrates in Hepatocellular Carcinoma
}

\author{
Yao Chen ${ }^{1 t}$, Haifeng Xie ${ }^{2 t}$, Ting Xie ${ }^{3}$, Xunjun Yang ${ }^{3,4}$, Yilin Pang ${ }^{3 *}$ and SongDao Ye ${ }^{4 *}$ \\ ${ }^{1}$ Department of Pathology, The First Affiliated Hospital of Wenzhou Medical University, Wenzhou, China, ${ }^{2}$ Hangzhou \\ Traditional Chinese Medicine (TCM) Hospital Affiliated to Zhejiang Chinese Medical University, Hangzhou, China, ${ }^{3}$ Zhejiang \\ Provincial Key Laboratory of Medical Genetics, Key Laboratory of Laboratory Medicine, Ministry of Education, School \\ of Laboratory Medicine and Life Sciences, Wenzhou Medical University, Wenzhou, China, ${ }^{4}$ Department of Laboratory \\ Medicine, The Second Affiliated Hospital and Yuying Children's Hospital of Wenzhou Medical University, Wenzhou, China
}

\section{OPEN ACCESS}

Edited by:

Shaoli Das,

National Institutes of Health $(\mathrm{NIH})$,

United States

Reviewed by:

Vishal Midya,

Icahn School of Medicine at Mount

Sinai, United States

Michael Poidinger,

Murdoch Childrens Research

Institute, Australia

*Correspondence:

Yilin Pang

ylpang2010@126.com

SongDao Ye

yesd955022@163.com

tThese authors have contributed equally to this work and share first

authorship

Specialty section:

This article was submitted to Computational Genomics, a section of the journal

Frontiers in Genetics

Received: 19 February 2021 Accepted: 30 April 2021 Published: 21 May 2021

Citation:

Chen Y, Xie H, Xie T, Yang $X$ Pang $Y$ and Ye $S$ (2021) Elevated Expression of PDZD11 Is Associated With Poor Prognosis and Immune

Infiltrates in Hepatocellular

Carcinoma. Front. Genet. 12:669928.

doi: 10.3389/fgene.2021.669928
Epithelial cells are held together by tight and adherent junctions, which are destroyed by the activation of epithelial-to-mesenchymal transition (EMT). The PLEKHA7-PDZD11 complex has been reported to be important for epithelial cell adhesion and connecting tissues. However, there is no research regarding the expression and role of PDZD11 in liver hepatocellular carcinoma (LIHC) progression. Here, we analyzed PDZD11 mRNA expression and its clinical results in LIHC patient RNA sequencing data based on different open databases. Furthermore, we examined differences in PDZD11 expression in LIHC tissues and cell lines using western blotting and real-time GPCR. These results are the first to report that the mRNA and protein levels of PDZD11 are significantly overexpressed in LIHC. Moreover, high expression of PDZD11 was correlated with poor overall survival in patients with LIHC. Gene regulatory network analysis suggested that PDZD11 is mainly involved in copper ion homeostasis, proteasome, and oxidative phosphorylation pathways. Interestingly, we found that PDZD11 levels were positively correlated with the abundance of immune infiltrates. In particular, higher infiltration levels of $\mathrm{CD} 4^{+} \mathrm{T}$ cells and macrophage subsets significantly affected $\mathrm{LIHC}$ patient prognosis. Taken together, these results demonstrate that PDZD11 could be a potential diagnostic and prognostic biomarker in LIHC.

Keywords: PDZD11, hepatocellular carcinoma, prognostic biomarker, immune infiltrates, functional network analysis

\section{INTRODUCTION}

Liver hepatocellular carcinoma (LIHC) accounts for the most common form of primary liver cancers (Villanueva, 2019), with an increasing incidence, particularly in East Asia (Bray et al., 2018; Siegel et al., 2019). LIHC is currently the third leading cause of cancer-related death worldwide (Jiang et al., 2019). LIHC likely occurs in patients with underlying liver diseases since infection with the hepatitis $\mathrm{B}$ or $\mathrm{C}$ virus ( $\mathrm{HBV}$ or $\mathrm{HCV}$ ) and long-term intoxication with alcohol or aflatoxin are the leading risk factors for developing LIHC (Jemal et al., 2017; Villanueva, 2019). Due to the high rate of recurrence and metastasis, the 5-year overall rate of survival for LIHC is only $18 \%$, making liver cancer the second-leading cause 
of cancer deaths, after pancreatic cancer (Jemal et al., 2017). Although operative treatment may be effective in the early stage of LIHC, the 5-year survival rate after developing to later stage is only $50-70 \%$ [European Association for the Study of the Liver (EASL), 2018]. Therefore, it is important to further screen LIHC oncogenes to help identifying novel biomarkers, therapeutic targets and immune-related biomarkers, and ultimately contribute to better diagnosis and prognosis of LIHC.

The human PDZD11 gene is located at chromosome Xq13.1 and is $3.92 \mathrm{~kb}$ long with 6 exons. The PDZD11 protein (140 aa) is a ubiquitously expressed small protein and mainly composed of a single PDZ domain (Stephenson et al., 2005). Previous studies have shown that diseases associated with PDZD11 include Purulent Acute Otitis Media and Middle Ear Disease (GeneCards database). PDZD11 was previously known as PISP, based on its interaction with the plasma membrane calcium ATPase (PMCA) b-splice variants, which may play a role in their sorting to or from the plasma membrane (Goellner et al., 2003). PDZD11 is also known as AIPP1, because it interacts with Menkes copper ATPase (ATP7A), which involves in maintaining copper homeostasis (Stephenson et al., 2005). Nabokina et al. (2011) demonstrated that PDZD11 interacted with human sodiumdependent multivitamin transporter (hSMVT) in intestinal epithelial cells and that this interaction affected biotin uptake process. Shah et al. (2016) reported that the interaction of the N-terminal region of PDZD11 with the WW1 domain of pleckstrin homology domain-containing A7 (PLEKHA7) was essential to stabilize junctional nectins at adherens junctions (AJ), and promote efficient junction assembly. Recent work has also shown that cooperative binding of the tandem WW domains (e.g., WW1 and WW2) of PLEKHA7 to PDZD11 promoted the binding of the C-terminus of Tspan33 to PLEKHA7. Furthermore, the complex formation of PLEKHA7, PDZD11, ADAM10 and its molecular chaperone Tspan33 through promoting the junctional clustering of the $\alpha$-toxin receptor ADAM10 makes cells more sensitive to the cytotoxic effects of Staphylococcus aureus $\alpha$-toxin (Vasileva et al., 2017; Rouaud et al., 2020).

Epithelial-to-mesenchymal transition (EMT) is a reversible cellular procedure that can transiently dedifferentiate epithelial cells into a mesenchymal phenotype (Dongre and Weinberg, 2019). Epithelial cells build strong connections with their neighbors and an apical-to-basal polarity via the sequential arrangement of adherens junctions, desmosomes, and tight junctions (Thiery et al., 2009). Conversely, EMT confers cells with invasive and metastatic potential, induces stem cell properties, inhibits apoptosis and senescence, and contributes to immunosuppression (Thiery et al., 2009). Therefore, EMT plays a crucial role in embryogenesis, wound-healing, organ fibrosis, tumor invasion and metastasis (Yan et al., 2018). In particular, about $90 \%$ of cancer-associated mortality is attributed to metastasis (Chaffer and Weinberg, 2011). Previous studies have shown that the combination of metastasis-related gene signatures and serum alpha-fetoprotein can be used as a good predictor of LIHC prognosis regardless of etiology and race (Yan et al., 2018).
The tumor microenvironment is composed of infiltrating inflammatory cells, stromal cells, and inflammatory mediators (Yan et al., 2018). Undoubtedly, the inflammatory microenvironment associated with hepatitis virus infection is an important factor influencing the invasion and metastasis of LIHC (Yan et al., 2018). Lara-Pezzi et al. (2001) also reported that hepatitis B virus HBx protein was able to induce adherens junction disruption in a src-dependent manner, which might contribute to the development of LIHC.

In this study, we first performed a bioinformatics analysis using different open databases to acquire detailed information about potential functions and prognostic value of PDZD11 in LIHC, and to explore whether the abnormal expression of PDZD11 is closely related to immune infiltrates of LIHC. Further, we verified the expression of PDZD11 in LIHC tissues, various human liver cancer cell lines and matched normal hepatocytes. The findings of this study may help us to understand the role of PDZD11 in the development of LIHC.

\section{MATERIALS AND METHODS}

\section{Patients and LIHC Tissue Specimens}

Seven pairs of matched LIHC tumor tissues and adjacent normal tissues of each pair of patients were immediately quenched in liquid nitrogen after surgical removal in the First Affiliated Hospital of Wenzhou Medical University. All the patients were clinically and pathologically confirmed as liver cancer. Informed consent was approved by the board of directors and the ethics committee of the First Affiliated Hospital of Wenzhou Medical University. Written informed consent was obtained from all subjects.

\section{Cell Culture}

HCCLM3, MHCC97H, HepG2, and L02 cells were cultured in high-glucose DMEM (GIBCO, Waltham, MA, United States) containing 10\% FBS (fetal bovine serum) (GIBCO, United States) and antibiotics $(100 \mathrm{U} / \mathrm{ml}$ penicillin and $100 \mu \mathrm{g} / \mathrm{ml}$ streptomycin) (GIBCO, United States), and incubated in an incubator containing $5 \% \mathrm{CO}_{2}$ at $37^{\circ} \mathrm{C}$.

\section{Western Blot Analysis}

Proteins in clinical tissues and whole-cells were extracted with $1 \%$ Triton X-100 lysis buffer supplemented with protease and phosphatase inhibitors (Sigma-Aldrich). Protein concentrations of the extracts were determined by the BCA assay kit (Thermo Fisher Scientific, Waltham, MA, United States). $40 \mu \mathrm{g}$ of total protein in each sample was separated by a $12 \%$ SDS-PAGE gels and transferred onto PVDF membrane (Bio-Rad, Hercules, CA) with a wet transfer system (Bio-Rad, United States). Block the blot in blocking buffer (5\% skim milk in TBST) on a shaker at room temperature for $1 \mathrm{~h}$, and then incubated with primary antibodies specific for PDZD11 (ab121210) (Abcam, Cambridge, MA) $(1: 2,000)$ and $\beta$-actin (Beyotime Biotechnology Co., Ltd., Shanghai, China) $(1: 5,000)$ overnight at $4^{\circ} \mathrm{C}$. The membrane was washed in TBST for $3 \times 15 \mathrm{~min}$ and then incubated with horseradish peroxidase (HRP)-conjugated anti-rabbit $(1: 5,000)$ 
and anti-mouse $(1: 20,000)$ immunoglobulin $G$ on a shaker at room temperature for $1.5 \mathrm{~h}$. Immunoreactive proteins were visualized using ECL reagent according to the manufacturer's protocol (Thermo Fisher Scientific, Rockford, IL). The optical density was quantified by executing ImageJ software.

\section{Quantitative RT-PCR}

LIHC cell lines and hepatocytes L-02 were seeded in $10 \mathrm{~cm}$ culture dish at a density of $2 \times 10^{6}$ cells per culture dish. After $36 \mathrm{~h}$ of incubation at $37^{\circ} \mathrm{C}$, cells were harvested and washed once with ice-cold PBS. The mRNA expression levels of genes were tested by SYBR green-based real-time quantitative PCR. Total RNA was extracted from all the cells using TRIzol reagent (Thermo Fisher Scientific, Waltham, MA, United States) according to the manufacturer's instructions. Total RNA ( $1 \mu \mathrm{g})$ was reverse-transcribed into cDNA ( + gDNA wiper) HiScript II Q Select RT SuperMix (Vazyme Biotech Co., Ltd., Nanjing, China) according to the manufacturer's instructions. The RT reaction was subsequently used as a template for real-time PCR. The reactions were performed on a CFX Connect ${ }^{\mathrm{TM}}$ Real-Time PCR Detection System (Bio-Rad, Hercules, CA) using ChamQ Universal SYBR qPCR Master Mix (Vazyme Biotech Co., Ltd., Nanjing, China). Primer sequences were as follows: PDZD11 5'CGGTGGTTTTCTTGCCTGCC3' (forward), 5'-TCAGTGTGATGGTTCGGGGC-3' (reverse) and $\beta$-actin $5^{\prime}$-AGCACAGAGCCTCGCCTTTG- ${ }^{\prime} \quad$ (forward), $5^{\prime}$-AAGCCGGCCTTGCACATG-3' (reverse). The PCR amplification procedures were as follows: pre-denaturation at $95^{\circ} \mathrm{C}$ for $3 \mathrm{~min}$, followed by 40 cycles of $\left(95^{\circ} \mathrm{C}\right.$ for $10 \mathrm{~s}$, $60^{\circ} \mathrm{C}$ for $30 \mathrm{~s}$ ). Record the threshold cycle number $(\mathrm{Ct})$ for each reaction. The $\mathrm{Ct}$ values of target genes were normalized to that of $\beta$-actin. Each sample was analyzed in triplicate and repeated 3 times.

\section{GEPIA2 Database}

The expression of PDZD11 mRNA in LIHC was analyzed using the GEPIA2 database ${ }^{1}$, which was developed by Peking University, China, and is based on The Cancer Genome Atlas (TCGA) and Genotype-Tissue Expression (GTEx) databases, including RNA sequencing and expression data from 33 malignant tumors, 8,587 normal tissues, and 9,736 tumor samples (Tang et al., 2017).

\section{Oncomine Analysis}

The Oncomine v.4.5 database ${ }^{2}$ is a comprehensive and userfriendly online cancer microarray database for DNA and RNA sequence analysis (Rhodes et al., 2007). In our study, mRNA expression levels and DNA copy number of PDZD11 in normal controls and cancer specimens were obtained from the Oncomine database. The retrieval conditions were as follows: analysis type/cancer vs. normal analysis, cancer type/liver cancer, dataset filters/data type/mRNA or DNA, and sample filters/sample type/clinical specification. The significance threshold was designed using the following specific parameters:

${ }^{1}$ http://gepia2.cancer-pku.cn/\#index

${ }^{2}$ https://www.oncomine.org/resource/login.html p-value of $1 \mathrm{E}-4$, -fold change of 2 , and gene rank in the top $10 \%$. Student's $t$-test was used to analyze differences in the expression of PDZD11 between normal controls and cancer specimens.

\section{DriverDBV3 Database}

DriverDBV3 $3^{3}$ uses a variety of -omics techniques to identify cancer driver genes and to present them with different molecular features, including somatic mutations, RNA expression, miRNA expression, DNA methylation, copy number variation, and clinical data, in addition to annotation of bases (Liu S.H. et al., 2020). The Gene Summary of PDZD11 in various cancer tissues and mRNA expression of PDZD11 in LIHC was analyzed using the DriverDBV3 database. Survival with a $\log$-rank $p<0.05$, was considered statistically significant.

\section{UALCAN Database Analysis}

The UALCAN database ${ }^{4}$ is a website for online analysis based on level 3 RNA-seq and clinical data of 31 cancer types from TCGA datasets (Chandrashekar et al., 2017). We used this database to analyze the differential expression and promoter methylation profile of PDZD11 in primary LIHC tissues and their association with clinicopathological parameters. Student's $t$-test was used to generate $p$-values; after Bonferroni correction for multiple measures, $p$ was still $<0.05$, which was statistically significant.

\section{cBioPortal Analysis}

The cBioPortal ${ }^{5}$ is an open-access web resource that provides visualization and analysis of multidimensional cancer genomics data (Gao et al., 2013). In this study, genetic alterations to PDZD11 in LIHC patients (TCGA, Firehose Legacy, 360 patients/samples) were investigated using the cBioPortal database.

\section{Protein-Protein Interaction (PPI) Network Analysis}

PPI network analysis of PDZD11 was conducted using the STRING ${ }^{6}$ (von Mering et al., 2003) and GeneMANIA ${ }^{7}$ (WardeFarley et al., 2010) online databases. We also used GeneMANIA to construct gene networks and predict the biological functions of gene sets in which Gene Set Enrichment Analysis (GSEA) was identified as being enriched in LIHC.

\section{LinkedOmics Database Analysis}

The LinkedOmics database ${ }^{8}$ (Vasaikar et al., 2018) is an online open-access powerful bioinformatics platform, which includes multi-omics information and clinical data involving 11,158 patients and 32 cancer types in the TCGA project. LinkedOmics was used to study genes differentially expressed in correlation with PDZD11 in LIHC. Pearson's correlation coefficient was applied to statistical analysis of the results produced by

\footnotetext{
${ }^{3}$ http://driverdb.tms.cmu.edu.tw/

${ }^{4}$ http://ualcan.path.uab.edu/

${ }^{5}$ https://www.cbioportal.org/

${ }^{6}$ https://string-db.org/

${ }^{7}$ http://genemania.org/

${ }^{8}$ http://www.linkedomics.org/login.php
} 
LinkedOmics. Then, genes positively and negatively correlated with PDZD11 in LIHC were selected based on the criteria of coefficient $>0.3$ and $<-0.3$. Finally, we enriched these gene sets by Gene Ontology (GO) analysis and Kyoto Encyclopedia of Genes and Genomes (KEGG) pathway enrichment analysis using the DAVID database ${ }^{9}$ (Huang da et al., 2009), and the results were visualized using an online platform ${ }^{10}$. Moreover, GSEA was utilized to perform various enrichment analyses, including for kinase targets, miRNA targets, and transcription factor targets. Ranking was based on the criteria of false discovery rate (FDR) $<0.05$, and 500 simulations were performed.

\section{TIMER Analysis}

Tumor Immune Estimation Resource (TIMER) ${ }^{11}$ is a comprehensive website for the systematic analysis of tumorinfiltrating immune cells ( $\mathrm{Li}$ et al., 2017). TIMER $2.0^{12}$ is the latest version of TIMER. We first analyzed the expression of PDZD11 in various tumors using the TIMER database, and the results were analyzed statistically using Wilcoxon rank sum test. Then, correlations between the expression of PDZD11 and the abundance of the six immune cell types (B cells, $\mathrm{CD} 8^{+} \mathrm{T}$ cells, $\mathrm{CD}^{+} \mathrm{T}$ cells, macrophages, neutrophils, and dendritic cells) in LIHC were analyzed using Spearman tests (tumor purity adjusted). Finally, the survival module was used to draw Kaplan-Meier plots for immune infiltrates and PDZD11 to determine survival differences. Statistical significance was set at $p<0.05$.

\section{Statistical Analysis}

GraphPad Prism (v.5.0) for Windows was used for statistical analysis, and $p<0.05$ was considered statistically significant. The log-rank test was used in Kaplan-Meier survival analysis. Student's $t$-test and Wilcoxon rank sum test were employed in two-group comparisons. Moreover, we conducted Bonferroni's correction for multiple measurements to ensure the credibility of multiple group comparisons. After Bonferroni correction, $p$ was still less than 0.05 , which represents a statistically significant difference.

\section{RESULTS}

\section{Elevated Expression of PDZD11 in LIHC}

To determine the differential expression of PDZD11 in diverse cancer types, PDZD11 mRNA expression was analyzed using the TIMER database. It was shown that the mRNA level of PDZD11 was significantly upregulated in bladder, breast, gallbladder, esophagus, kidney, liver, lung, gastric, thyroid, and uterine corpus endometrial carcinoma (Figure 1A). Further analysis showed that PDZD11 was overexpressed in LIHC patients in the GEPIA2 database (Figure 1B). In the ONCOMINE database, PDZD11 was also identified with significantly higher levels in

\footnotetext{
${ }^{9}$ https://david.ncifcrf.gov/home.jsp

${ }^{10}$ http://www.bioinformatics.com.cn/

${ }^{11}$ https://cistrome.shinyapps.io/timer/

${ }^{12}$ http://timer.cistrome.org/
}

LIHC in multiple datasets. In the Chen Liver dataset, PDZD11 overexpression was found in LIHC tissues compared with normal tissues with a -fold change of $1.812(p=3.44 \mathrm{E}-22)$, while Wurmbach observed a 1.651-fold increase in PDZD11 mRNA expression in LIHC samples $(p=8.16 \mathrm{E}-5)$ (Figures 1 C,D). In addition, we analyzed PDZD11 expression using the DriverDBV3 database. We found that the results were largely consistent with those in the ONCOMINE database. However, there was no statistically significant difference in the mRNA expression of PDZD11 observed in recurrent solid tumors compared to adjacent normal liver tissues (Figure 1E). Consistently, protein analysis involving eight patients (including eight tumor tissue and eight matched adjacent normal tissues) diagnosed with liver cancer confirmed that PDZD11 abundance was elevated in LIHC tissues (Figure 1F). Additionally, we found increased levels of PDZD11 in LIHC cell lines at both the mRNA and protein levels (Figures 1G,H) compared to that found in normal human L02 hepatocytes. However, the protein expression of PDZD11 in HepG2 cells was significantly lower than that in L-02 cells.

\section{Relationship Between PDZD11 mRNA Levels and Clinicopathological Parameters in LIHC Patients}

Next, relationships between PDZD11 mRNA expression and clinicopathological parameters of LIHC patients were analyzed using the UALCAN database. The results showed that PDZD11 was upregulated in primary LIHC tissues compared to adjacent normal tissues (Figure 2A, $p<0.001$ ). As shown in Figures 2BI, according to subgroup analysis based on race, gender, age, weight, and lymph node metastasis status, the mRNA expression of PDZD11 in LIHC patients was evidently higher than that in healthy individuals. In particular, the expression of PDZD11 mRNA was clearly correlated with more advanced and lessdifferentiated tumors in LIHC patients, who tended to express higher PDZD11 mRNA levels. The highest mRNA expression of PDZD11 was found in stage 3 and/or tumor grade 3 cases (Figures 2B,G). The reason why mRNA expression of PDZD11 in stage 3 and/or tumor grade 3 seemed to be higher than that in stage 4 and tumor grade 4 may be due to the small number of samples. In addition, PDZD11 mRNA expression was positively correlated with TP53 mutation status, and was also significantly elevated in LIHC patients with TP53 mutations (Figure 2I).

\section{Frequency and Types of PDZD11 Alterations in LIHC}

Genetic alterations to PDZD11 in LIHC were evaluated using the cBioPortal database. As shown in Figure 3A, among the 360 LIHC patients sequenced, 27 showed genetic alterations, with a mutation rate of $8 \%$. Moreover, we observed that mRNA upregulation was the only aberrant type of genetic alteration involving PDZD11 in LIHC.

\section{Decreased PDZD11 Promoter Methylation Levels in LIHC}

To analyze why expression of PDZD11 mRNA was significantly higher in LIHC tissues than in adjacent normal liver tissues, we 


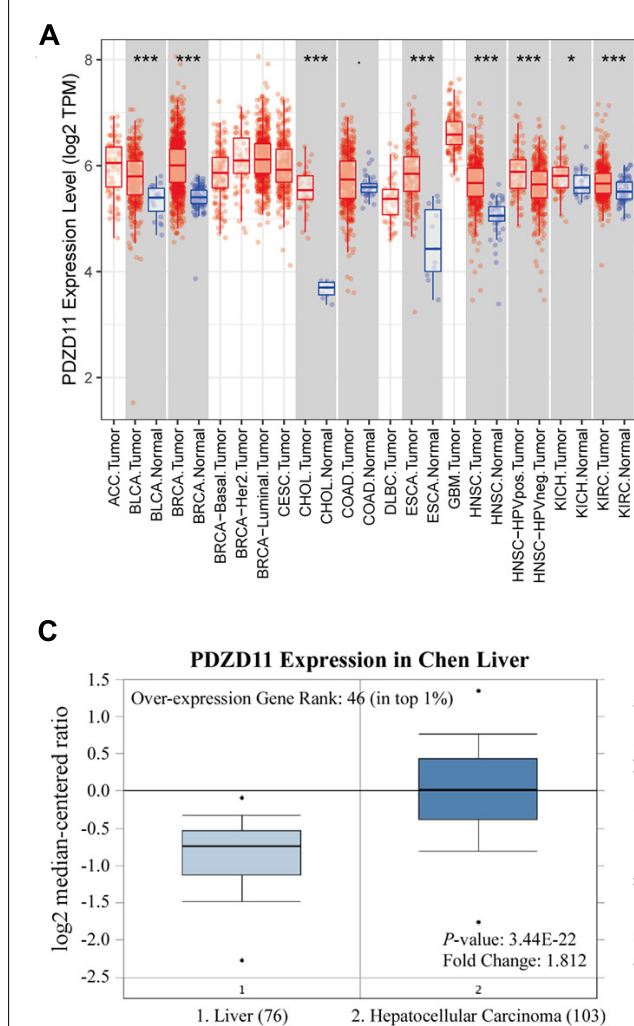

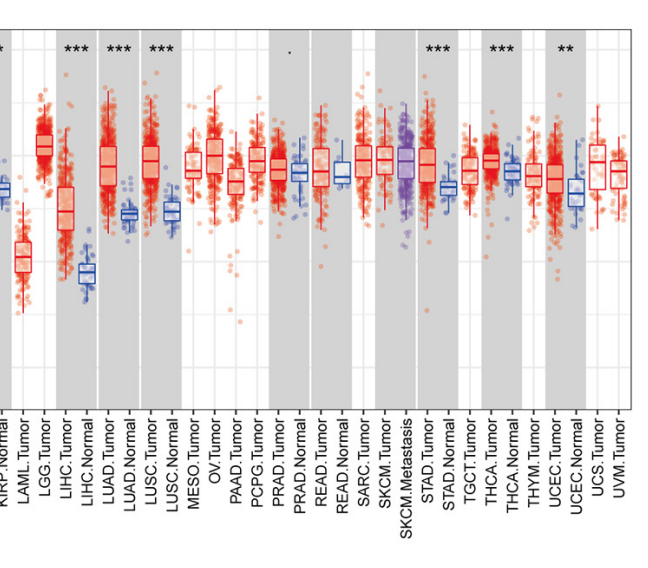

D

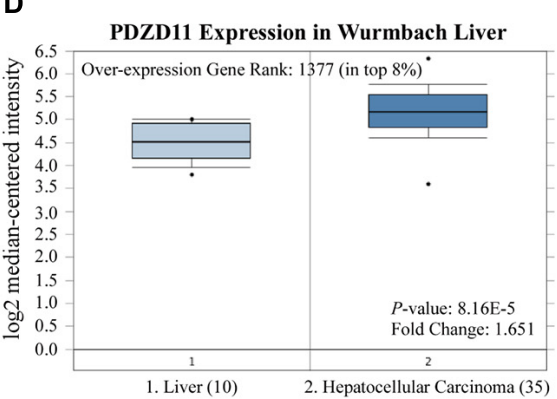

E
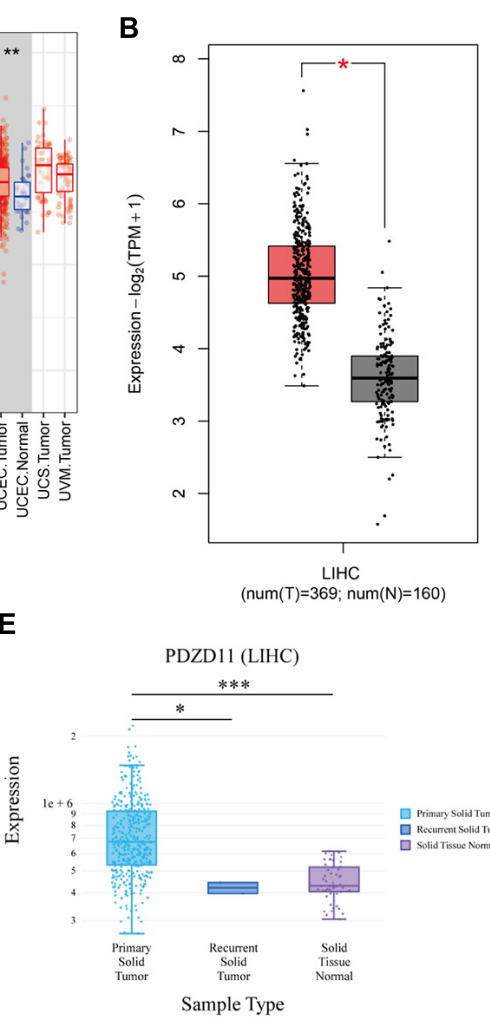

$\mathbf{F}$

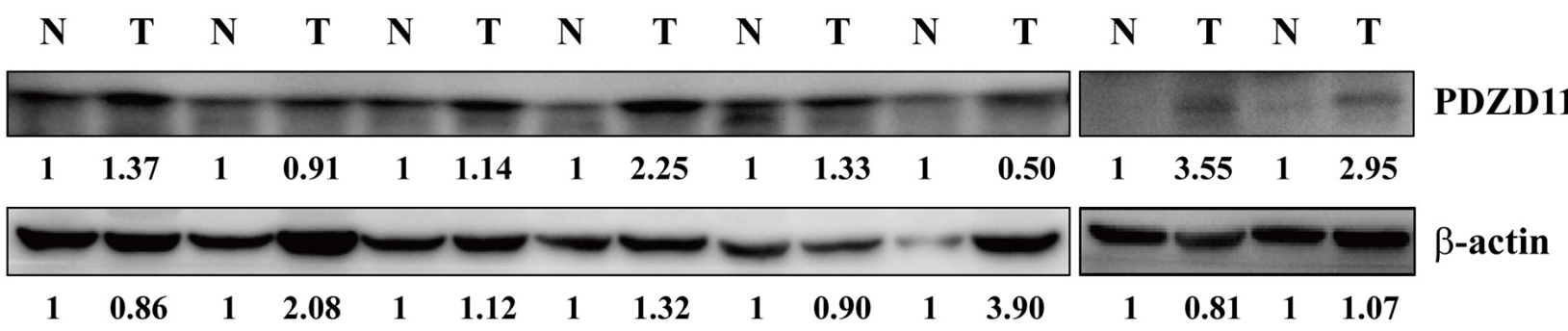

G

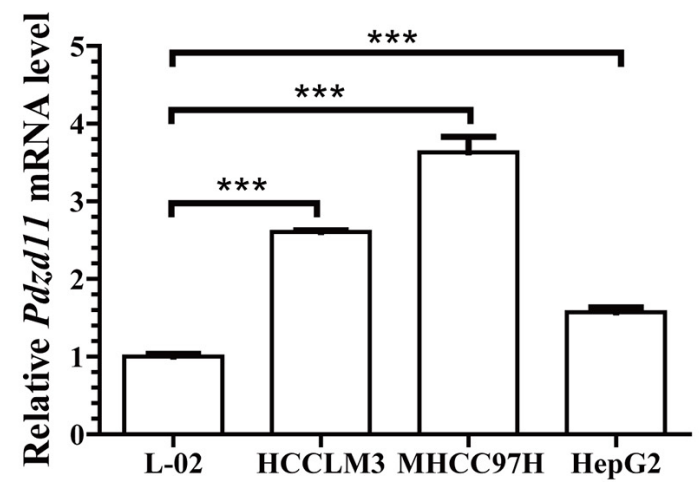

H

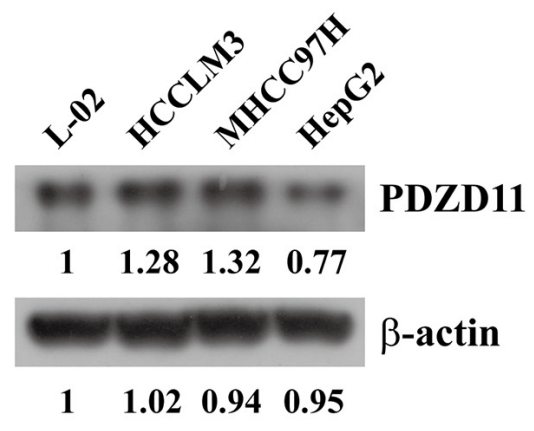

FIGURE 1 | PDZD11 expression levels in LIHC. (A) Transcription levels of PDZD11 in different types of cancers (TIMER database). (B) PDZD11 mRNA expression levels in LIHC tissues and adjacent normal liver tissues from GEPIA 2 database. (C,D) Box plots show PDZD11 mRNA expression in liver (left plot) and hepatocellular carcinoma tissue (right plot) of the Chen Liver (C) and Wurmbach Liver (D) datasets. The fold-change of PDZD11 expression in LIHC was determined using the Oncomine database. The threshold was designed using the following specific parameters: $p=1 \mathrm{E}-4$, fold change $=2$, and gene rank $10 \%$. (E) $\mathrm{mRNA}$ expression of PDZD11 in primary solid tumors, recurrent solid tumors, and adjacent normal liver tissues (DriverDBV3 database). (F) A representative western blot showing PDZD11 protein is expressed in LIHC tissues (T) and matched normal liver tissues $(\mathrm{N})(n=8)$. (G,H) Real-time qPCR and Western blotting analysis of PDZD11 mRNA (G) and protein expression $\mathbf{( H )}$ in human hepatocytes and LIHC cell lines. ${ }^{*} p<0.05 ;{ }^{* \star} p<0.01 ;{ }^{* \star *} p<0.001$. 


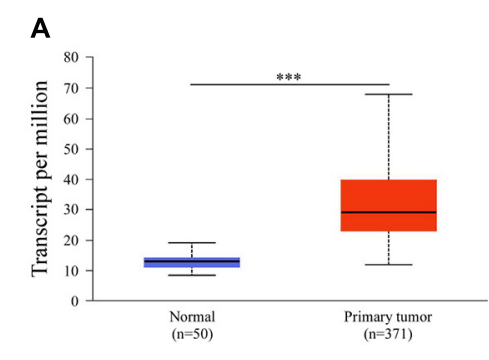

D

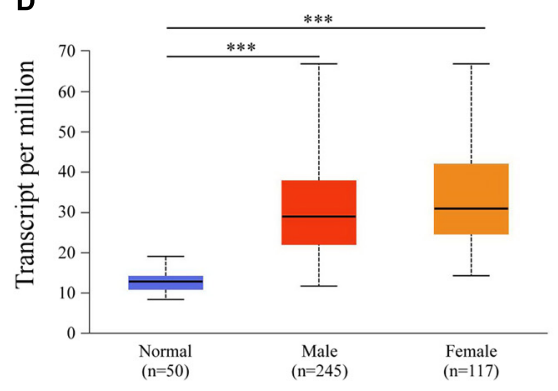

G

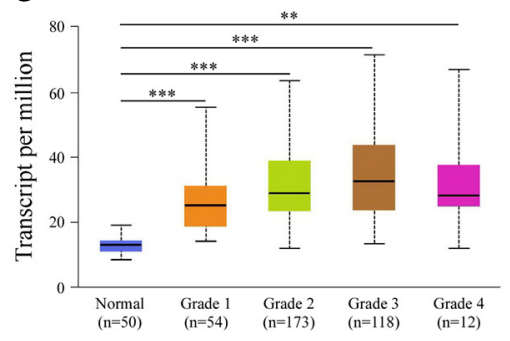

B

H

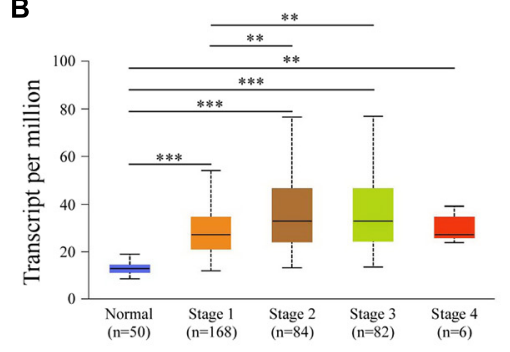

E
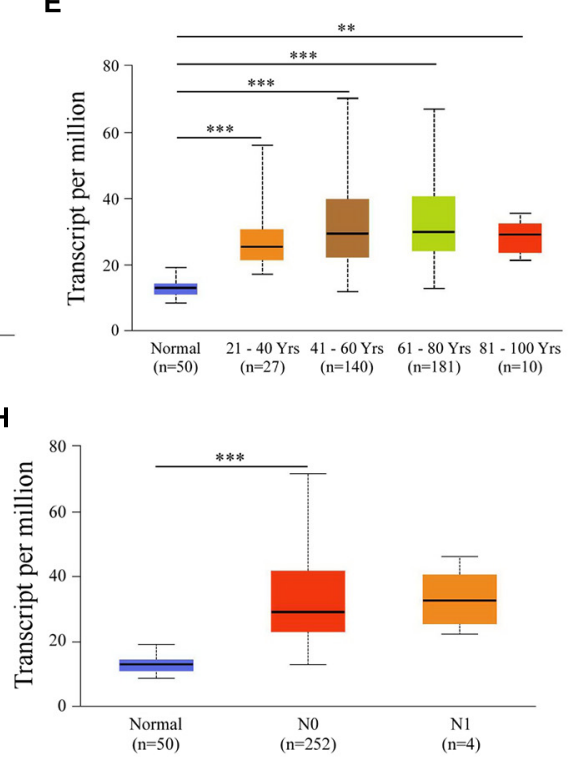

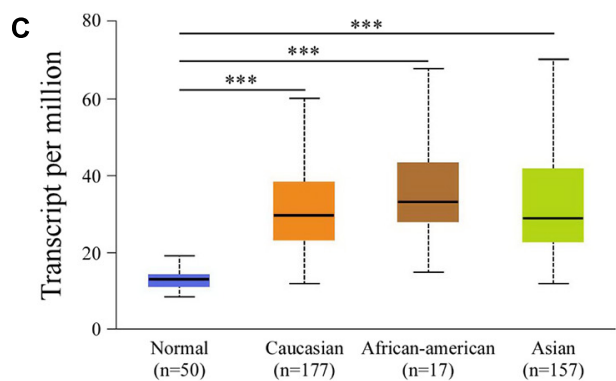

$\mathbf{F}$
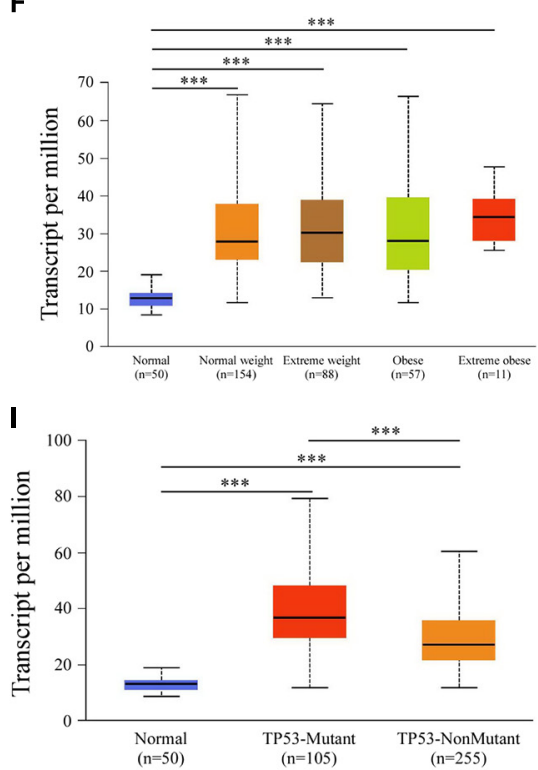

FIGURE 2 | Analysis of subgroup expression of PDZD11 in LIHC (UALCAN database). (A) PDZD11 mRNA expression in LIHC tissue and adjacent normal liver tissue. (B-I) Box plot shows the PDZD11 mRNA expression of LIHC patients in the subgroups of different cancer stages (B), race (C), gender (D), age (E), weight (F), tumor grade (G), nodal metastasis status (H), and TP53 mutation status (I). The data are shown as mean \pm SE. ${ }^{*} p<0.05 ;{ }^{* *} p<0.01 ;{ }^{* * *} p<0.001$. The asterisk indicates a significant difference between the two sets of data.

used the UALCAN database to evaluate the extent of PDZD11 promoter methylation in LIHC samples and investigated the association between promoter DNA methylation and PDZD11 expression levels. The results indicated that PDZD11 promoter methylation levels were lower in LIHC cases than in normal control samples (Figure 3B). To explore the factors that affect levels of PDZD11 promoter methylation, we further analyzed promoter DNA methylation of PDZD11 in different subgroups according to different clinicopathological parameters. The subgroup analysis results showed that promoter methylation of PDZD11 was possibly affected by individual cancer stages, race, gender, age, weight, tumor grade, nodal metastasis status, and TP53 mutation status in LIHC (Figures 3C-J).

\section{Prognostic Value of PDZD11 mRNA Expression in LIHC Patients}

To explore whether high expression levels of PDZD11 are associated with cancer-promoting or tumor suppressor genes, we evaluated the prognostic value of PDZD11 mRNA expression in patients with LIHC using the DriverDBV3 database. As shown in Figures 4A,B, PDZD11 overexpression was associated with unfavorable 5-year survival [hazard ratio $(\mathrm{HR})=1.69$, log-rank $p=0.0036]$ and overall survival (OS, $\mathrm{HR}=1.53$, log-rank $p=0.0153)$ in LIHC patients.

\section{Biological Interaction Network of PDZD11}

Using the STRING and GeneMANIA databases, a functional protein interaction network of PDZD11 was constructed to enrich for possible PDZD11-mediated signaling pathways (Figures 4C,D). ATP7A, a transmembrane protein that functions in copper transport across cell membranes, was the only gene that intersected two protein-protein interaction (PPI) networks (Schmidt et al., 2018). Furthermore, STRING was used to perform GO and KEGG analyses to determine the functional enrichment of these 29 interactors. The results indicated that biological processes included copper ion homeostasis and copper ion transmembrane transport; Cellular components analysis found that these proteins are localized mainly in endosomes and early endosomes. Molecular function analysis indicated that these 


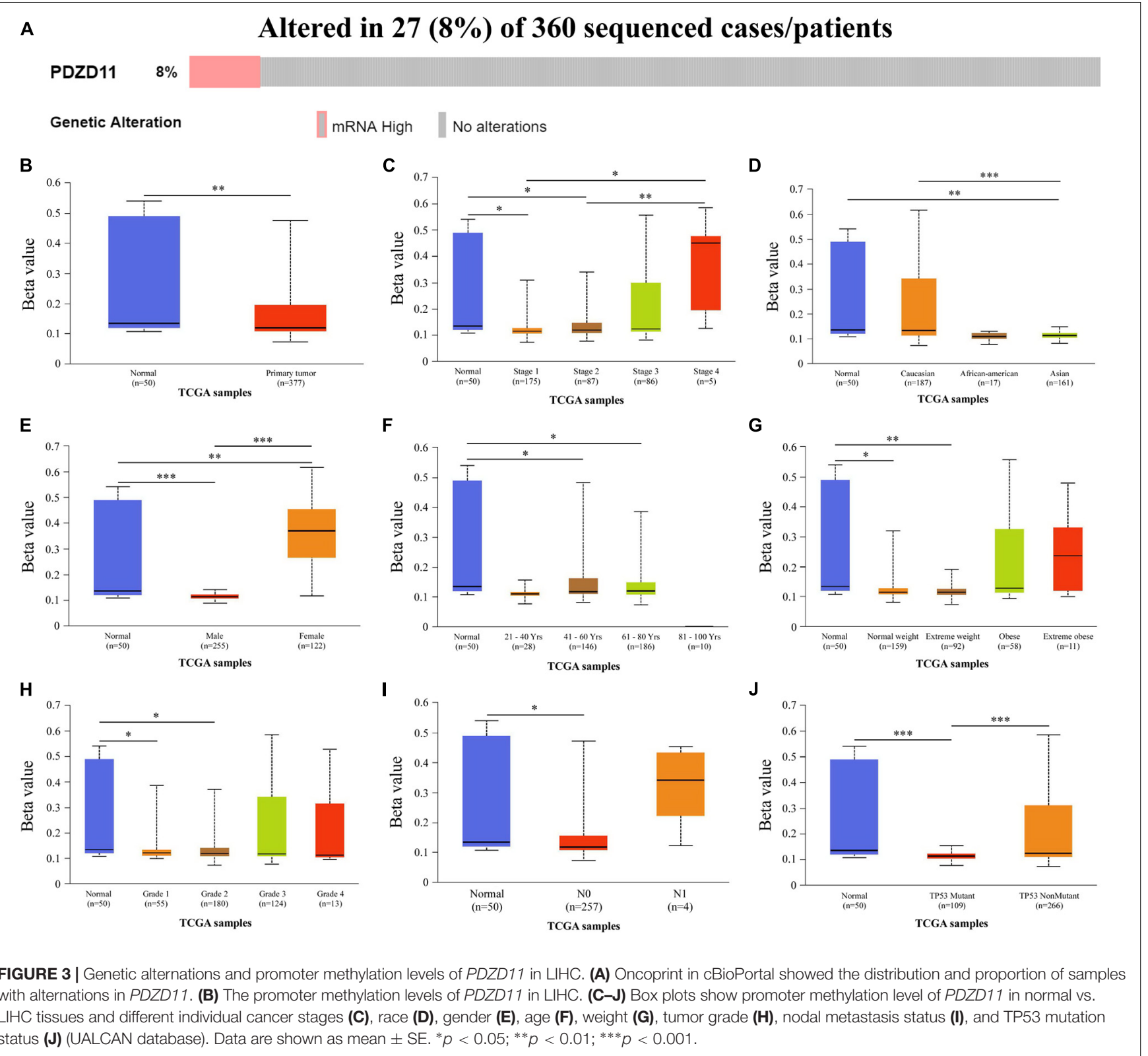

proteins are primarily involved in copper-dependent protein binding, copper ion transmembrane transporter activity, copper ion binding, copper chaperone activity and phosphatidylinositol3,5-bisphosphate binding (data not shown).

\section{Enrichment Analysis of PDZD11 Functional Networks in LIHC}

Predicted Functions and Pathways of Co-expressed Genes Correlate With PDZD11 in LIHC

LinkedOmics was used to analyze TCGA mRNA sequencing data from 371 LIHC patients. Pearson's test was used to analyze the co-expression of genes correlated with PDZD11 levels in LIHC. As shown in the volcano plot (Figure 5A), 2,960 genes (dark red dots) showed significant positive correlation with PDZD11, whereas 3,234 genes (dark green dots) showed opposite correlations (false discovery rate $($ FDR $)<0.01)$. The top 50 significant genes were positively and negatively associated with PDZD11, as shown in the heat map (Figures 5B,C). As shown in Figures 5D-F, the mRNA expression of PDZD11 showed the strongest positive association with expression of FAM50A (Pearson correlation $=0.62, p=7.71 \mathrm{e}-41)$, NDUFA1 (Pearson correlation $=0.60, p=9.78 \mathrm{e}-38$ ), and LAGE3 (Pearson correlation $=0.60, p=1.04 \mathrm{e}-37)$, which reflect changes in the spliceosome complex (Lee et al., 2020), mitochondrial respiratory chain complex I (Fernandez-Moreira et al., 2007), and the KEOPS/EKC complex (tRNA modification complex) (Wan et al., 2017).

Furthermore, based on the results of the Pearson test (Figures 5A-C), we selected positively and negatively correlated 


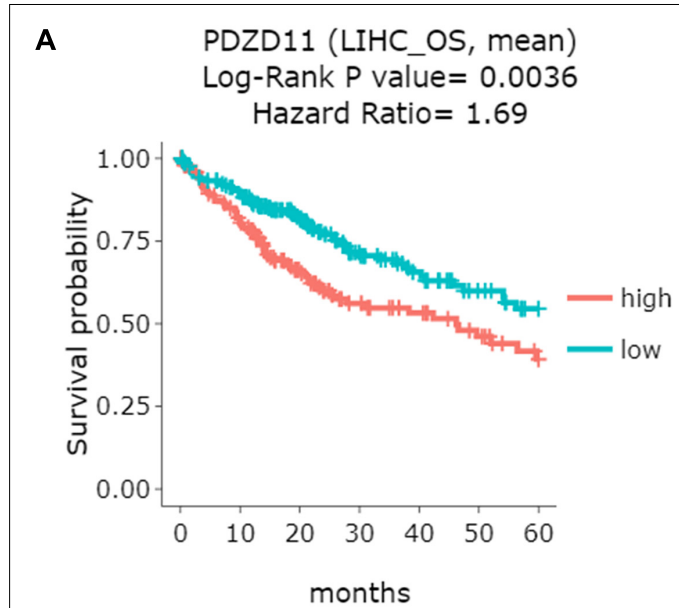

C

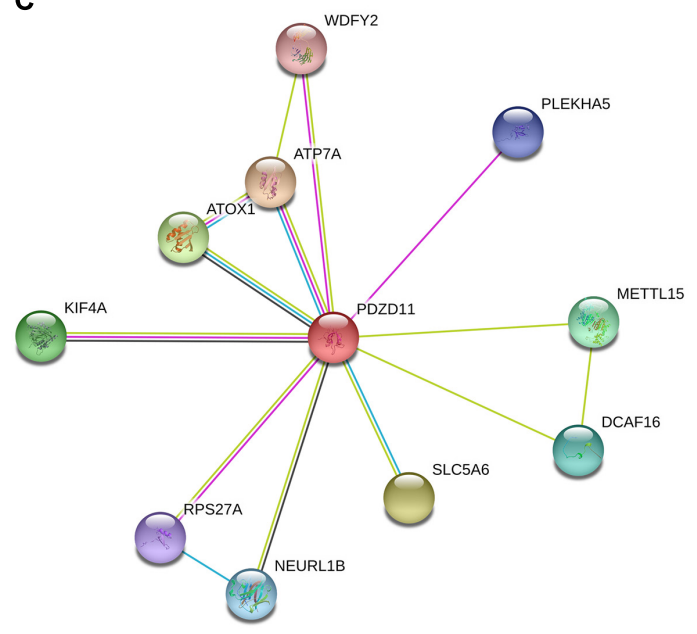

B

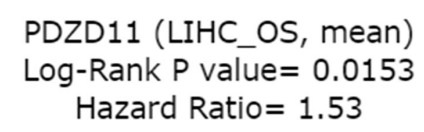

Hazard Ratio $=1.53$

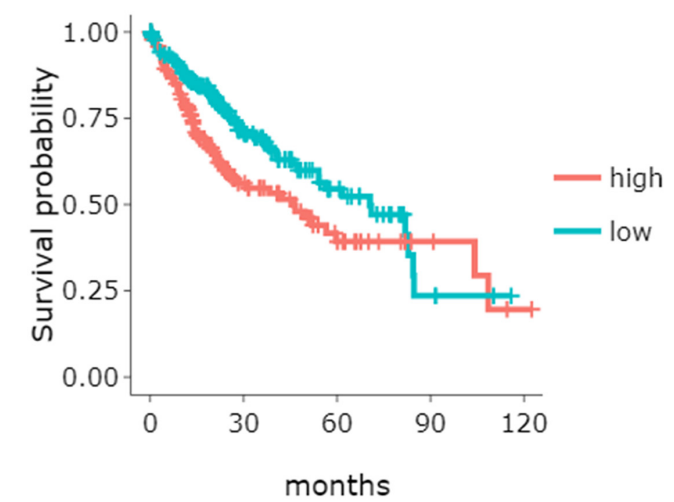

D

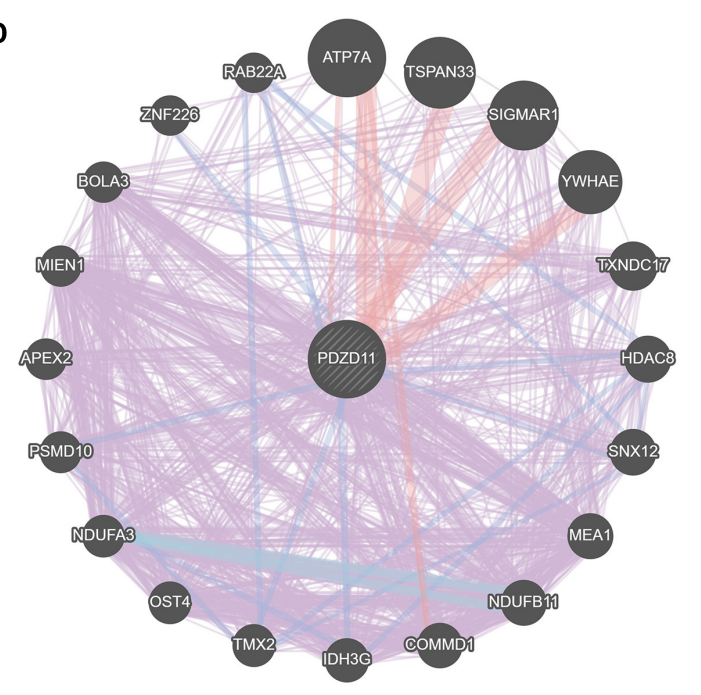

Networks

- Physical Interactions

1- Co-expression

-1 Predicted

- Co-localization

-1 Pathway

FIGURE 4 | Visual summary of the prognostic value and biological interaction network of PDZD11 in LIHC. (A,B) Kaplan-Meier plot of the relationship of PDZD11 gene expression and survival in LIHC patients (DriverDBV3 database). The LIHC patient samples are stratified into 2 groups using the mean expression value as the cut-off point: 149 samples with highly expressed PDZD11 mRNA (red) and 216 samples with lowly expressed PDZD11 mRNA (green). $Y$-axis is survival probability. The left figure is the 5-year survival, and the right figure shows overall survival (OS). The $X$-axis is the months of survival period. Log-Rank $P$-value and Hazard Ration (HR) are provided on the top of plots. (C) Protein-protein interaction (PPI) network of PDZD11 (Top 10) (STRING database). (D) PPI network (Top 20) of PDZD11 from GeneMANIA database.

genes with coefficients $>0.3$ and $<-0.3$. Finally, 617 genes positively correlated with PDZD11 and 411 genes negatively correlated with PDZD11 were selected (FDR $<0.001$ ). Moreover, these genes were used for GO and KEGG enrichment analyses using the DAVID database. The cutoff criterion was set at FDR $<0.01$. As shown in Figures 6A-D, cellular component analysis indicated that these proteins were mainly located in the nucleoplasm, proteasome complex, and mitochondrial inner membrane. Biological processes were primarily enriched in NIK/NF- $\mathrm{BB}$ signaling, regulation of cellular amino acid metabolic processes, and anaphase-promoting complex-dependent catabolic processes. Molecular function analysis revealed that these proteins were mostly involved in protein binding, poly(A) RNA binding, and threonine-type endopeptidase activity. KEGG pathway results showed that the co-expressed genes for the most part participated in proteasomes, oxidative phosphorylation (OXPHOS) (Figure 6E), and Alzheimer's disease.

\section{PDZD11 Networks of Kinase, MicroRNA or Transcription Factor Targets in LIHC}

To further explore the gene regulatory network of PDZD11 in LIHC, we also analyzed the important kinase, miRNA, and transcription factor target networks that were connected to PDZD11 in LIHC via gene set enrichment analysis (GSEA). The results showed that the most frequent kinase targets, miRNA targets, and transcription factor targets were kinase CDK5, three miR-200 family members (miR-200b, miR-200c, and miR-429), 


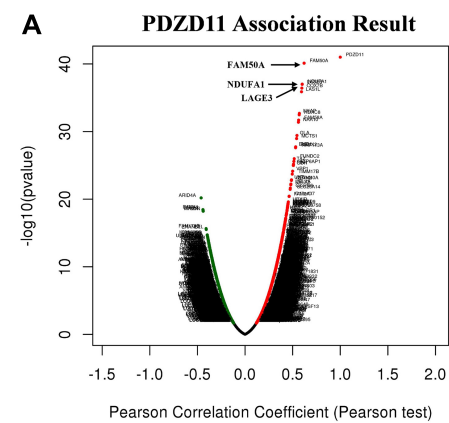

D

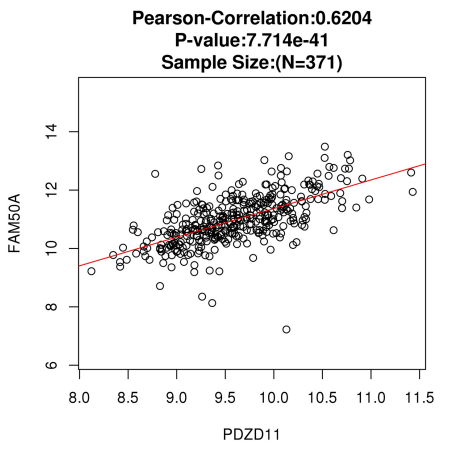

B Positively correlated with PDZD11 in LIHC

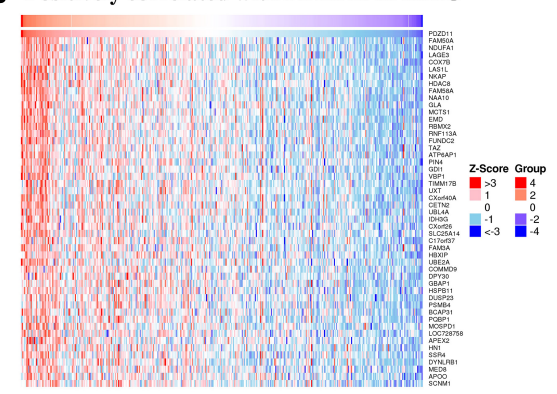

E

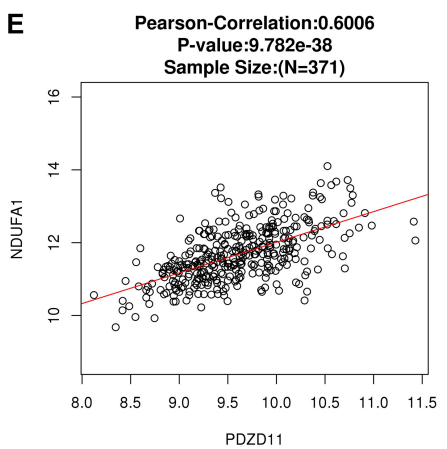

C Negatively correlated with PDZD11 in LIHC

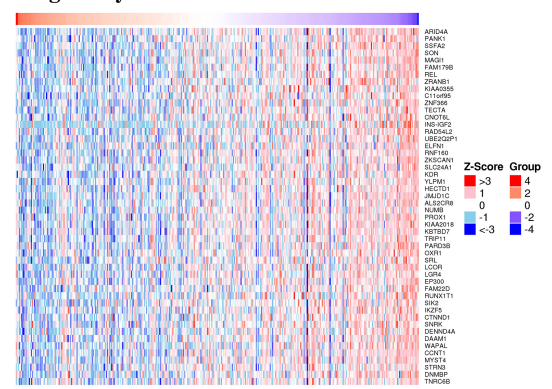

F Pearson-Correlation:0.6004
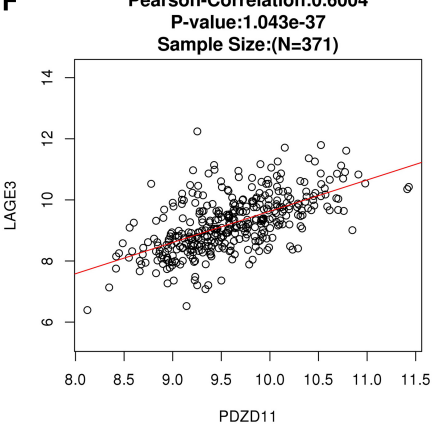

FIGURE 5 | Differentially expressed genes that correlated with PDZD11 in LIHC (LinkedOmics database). (A) A Pearson test was used to determine the correlations between PDZD11 and differently expressed genes in LIHC. (B,C) Heat maps are showing genes (Top 50) positively or negatively correlated with PDZD11 in LIHC. Red indicates positively correlated genes and blue indicates negatively correlated genes. (D-F) The scatter plots mean that PDZD11 expression is positively correlated with the expression of FAM5OA (D), NDUFA1 (E), and LAGE3 (F).

and V\$SOX9_B1, respectively (Table 1 and Supplementary Tables 5-7). Furthermore, PPI networks were constructed by STRING, and biological enrichment was performed using the DAVID database, indicating that all three gene sets were mainly involved in the KEGG pathway of prostate cancer, MAPK signaling pathway, and transcriptional dysregulation in cancer (Supplementary Figures 1-3).

\section{Association of PDZD11 Expression and Immune Infiltration in LIHC}

LIHC is one of the most common malignant tumors (Okajima et al., 2017). Because PDZD11 overexpression is associated with poor prognosis in LIHC patients (Figure 4), we explored whether the expression of PDZD11 was correlated with levels of immune infiltration in LIHC from the TIMER database and/or TIMER2.0 database. As shown in Figure 7, there was a positive correlation between PDZD11 expression and infiltration by $\mathrm{B}$ cells, $\mathrm{CD} 8^{+} \mathrm{T}$ cells, $\mathrm{CD} 4^{+} \mathrm{T}$ cells, macrophages, neutrophils, and dendritic cells. Furthermore, under the premise of high expression of PDZD11 mRNA in LIHC, we found that higher infiltration levels of two immune cells ( $\mathrm{T}$ cell CD4 + memory resting-CIBERSORT, and Macrophage-EPIC) were associated with better survival outcomes in LIHC patients (Figures 7B,C). In contrast, higher infiltrating levels of the macrophage M2 subset was a risk factor for disease prognosis in LIHC patients (Figure 7D).

\section{DISCUSSION}

EMT-induced changes in epithelial cell plasticity are evidenced by the loss of epithelial markers, such as the adherence junction component E-cadherin and cytokeratins of the intermediate filament system (K8, K18, K19). Conversely, the expression of mesenchymal proteins such as $\mathrm{N}$-cadherin, $\alpha$-SMA, FSP-1, and the EMT transcription factors Snail (SNA1), Slug (SNA2), Twist, and ZEB are increased (Giannelli et al., 2016). Konopka et al. (2007) have also reported that junctional adhesion moleculeA (JAM-A) is critical for the formation of pseudocanaliculi and regulates E-cadherin expression through feedback signaling pathways in hepatic cells. However, the present study lacked a well-defined consensus on EMT-MET (mesenchymal-epithelial transition) biomarkers, which hinders definitive conclusions on how EMT affects clinical outcomes in LIHC patients (Giannelli et al., 2016). Therefore, there is an urgent need to identify biomarkers or therapeutic targets related to EMT for early diagnosis and for predicting the progression and recurrence of LIHC.

Current research reports that the interaction of PDZD11 with PLEKHA7 is significantly associated with tight and adherens junctions (Guerrera et al., 2016; Vasileva et al., 2017). However, to the best of our knowledge, no study has investigated the role of PDZD11 in liver cancer. In this study, we provide the first evidence that PDZD11 mRNA expression is significantly upregulated in LIHC and is associated with poor prognosis 


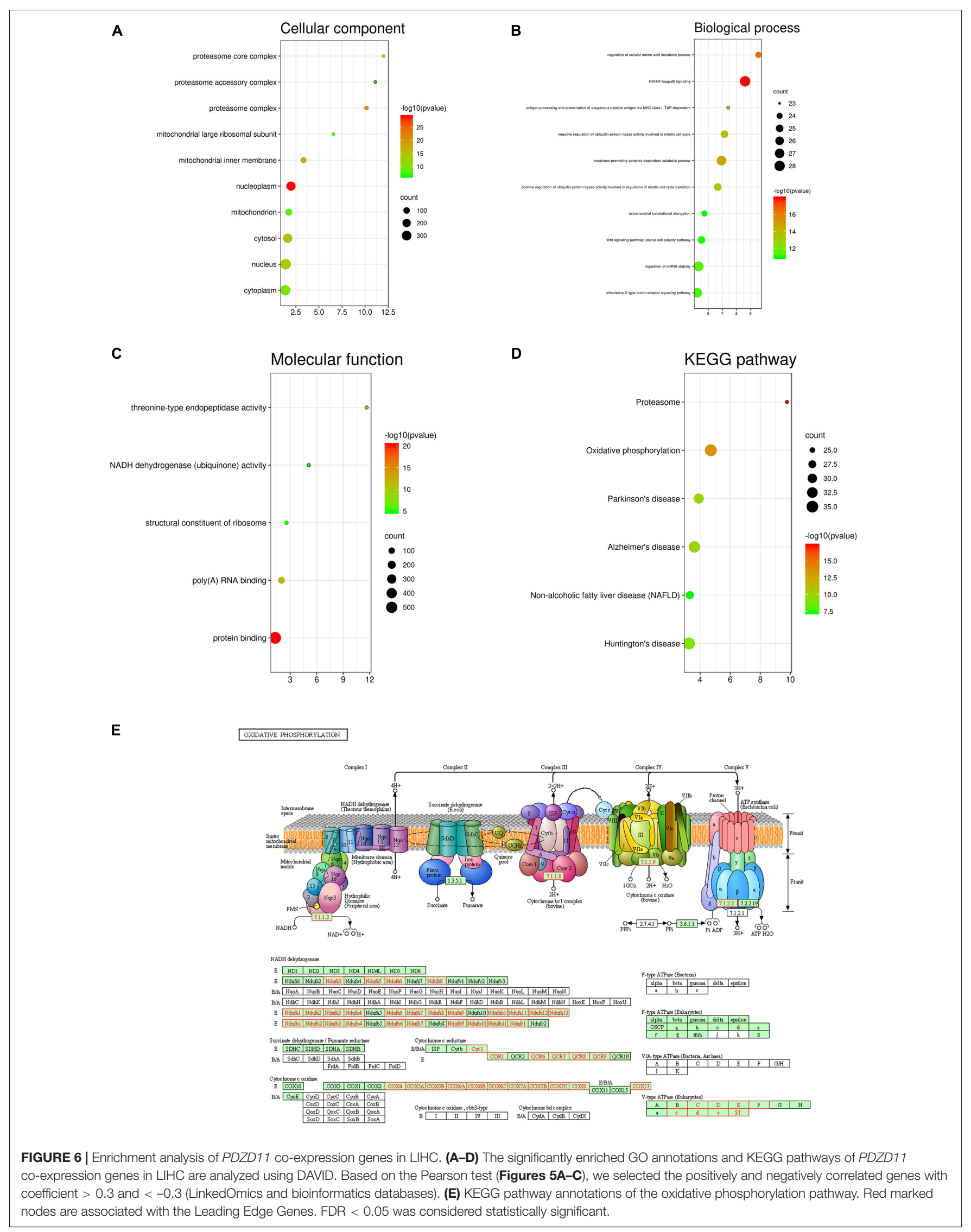


TABLE 1 | Kinase, miRNA and transcription factor-target networks of PDZD11 in LIHC (LinkedOmics).

\begin{tabular}{|c|c|c|c|c|}
\hline Enriched category & Geneset & Leading edge number & FDR & $P$-value \\
\hline \multirow[t]{4}{*}{ Kinase target } & Kinase_CDK5 & 26 & 0.0066696 & 0 \\
\hline & Kinase_NLK & 5 & 0.010671 & 0 \\
\hline & Kinase_MAPK7 & 14 & 0.034682 & 0 \\
\hline & Kinase_DYRK1A & 7 & 0.045353 & 0.0040650 \\
\hline \multirow[t]{5}{*}{ miRNA target } & GTGTTGA,MIR-505 & 46 & 0 & 0 \\
\hline & CAGTATT,MIR-200B,MIR-200C,MIR-429 & 155 & 0 & 0 \\
\hline & ACTGAAA,MIR-30A-3P,MIR-30E-3P & 81 & 0 & 0 \\
\hline & AAAGGGA,MIR-204,MIR-211 & 101 & 0 & 0 \\
\hline & TACTTGA,MIR-26A,MIR-26B & 131 & 0 & 0 \\
\hline \multirow[t]{5}{*}{ Transcription factor target } & GGAANCGGAANY_UNKNOWN & 35 & 0 & 0 \\
\hline & V\$FREAC4_01 & 49 & 0 & 0 \\
\hline & V\$HOX13_01 & 15 & 0 & 0 \\
\hline & V\$SOX9_B1 & 80 & 0 & 0 \\
\hline & V\$STAT5A_02 & 50 & 0 & 0 \\
\hline
\end{tabular}

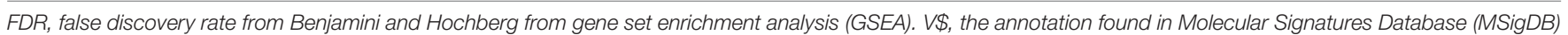
for transcription factors (TF).

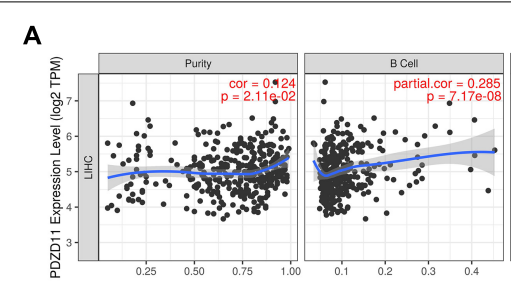

B

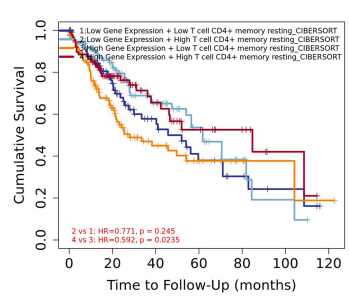

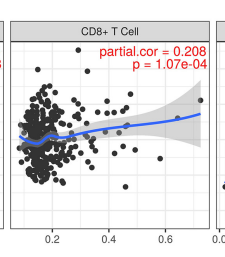

C
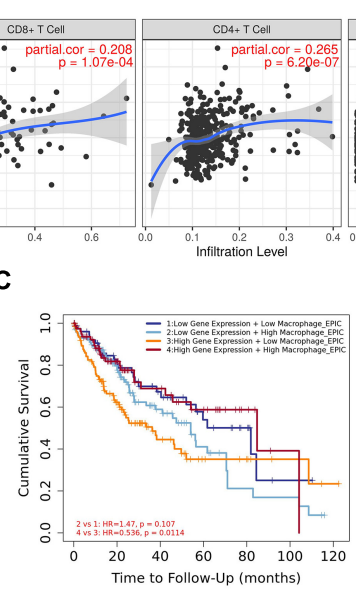
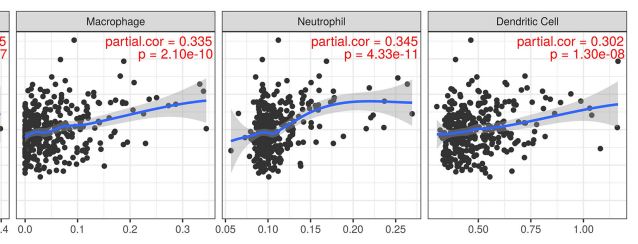

D

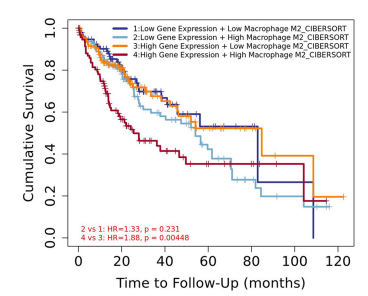

FIGURE 7 | Associations between mRNA expression of PDZD11 and immune infiltration in LIHC (TIMER2.0 database). (A) Association of PDZD11 expression with abundance of immune infiltrates (B cells, CD8 ${ }^{+} \mathrm{T}$ cells, CD4 ${ }^{+} \mathrm{T}$ cells, macrophages, neutrophils and dendritic cells). (B-D) The effect of PDZD11 expression in correlation with infiltration levels of immune cells on the prognosis of $\mathrm{LIHC}$.

(Figures 1A-E, 4A,B). In particular, we demonstrated that PDZD11 is aberrantly expressed in human liver cancer tissues and cell lines (Figures $\mathbf{1 F}-\mathbf{H}$ ). Moreover, subgroup analysis showed that the mRNA expression of PDZD11 was also upregulated in different subgroups of LIHC (Figure 2). In particular, the mRNA expression of PDZD11 increased as tumors progressed (Figures 2B,G). Additionally, we found that the expression of PDZD11 may be negatively regulated by wildtype p53 at the transcriptional level (Figure 2I). Similarly, previous studies have shown that E-cadherin, the most reliable and closely investigated marker in a large number of LIHC patients, was directly correlated with poorer prognosis and shorter survival (Yamada et al., 2014). Consequently, these results suggest that PDZD11 and the EMT marker E-cadherin could serve as potential diagnostic and prognostic biomarkers in LIHC patients.
mRNA upregulation is the most aberrant type of genetic alteration involving PDZD11 in LIHC (Figure 3A). We further analyzed PDZD11 promoter DNA methylation levels and found that the higher expression of PDZD11 in LIHC may be negatively correlated with the extent of promoter methylation (Figure 3B). Subgroup analysis showed that PDZD11 promoter methylation level was also downregulated in different subgroups of LIHC (Figures 3C-J). Cano et al. (2000) reported that the expression of the EMT marker E-cadherin is negatively regulated by the transcription factor Snail. These results suggest that the mechanism of high expression of PDZD11 mRNA in LIHC could be different from the classical Snail/E-cadherin axis.

Further analysis of the gene regulatory network of PDZD11 in LIHC suggested that the functions of these genes were primarily related to copper ion homeostasis, proteasome, and OXPHOS pathway. As shown in Figures 4C,D, ATP7A is the 
only gene that intersects the two PPI networks. A previous study demonstrated that ATP7A is a transmembrane protein that functions in copper transport across cell membranes (Schmidt et al., 2018). Bortezomib is a first-in-class proteasome inhibitor that has been repeatedly demonstrated to exert antiproliferative, anti-metastatic, and pro-apoptotic effects in LIHC (Yang et al., 2016; Huang et al., 2019). This study showed that the protein expression level of PDZD11 was irreconcilable with its mRNA transcription level. However, this proteasomemediated PDZD11 protein degradation pathway requires further research. A recent study has also reported that induced E-cadherin expression and subsequent induction of NF- $\mathrm{B}$ signaling increases OXPHOS, glycolysis, and cell proliferation in human gastric adenocarcinomacells (Park et al., 2017). Therefore, further research is needed to determine how abnormal expression of PDZD11 affects OXPHOS in LIHC and its role in LIHC metastasis.

We also sought important networks of target kinases, miRNAs, and transcription factors of the differentially expressed PDZD11 in LIHC. We found that PDZD11 in LIHC was linked to a network of kinases, including CDK5, NLK, and MAPK7. Previous studies have reported that levels of these kinases are significantly higher in human LIHC tissue than in normal liver tissue. Moreover, the downregulated expression of these kinases significantly inhibits the development and growth of LIHC in vitro and in vivo (Jung et al., 2010; Ehrlich et al., 2015; Lu et al., 2016). The probable miRNAs involved in the regulation of PDZD11 expression in LIHC included miR-505, three miR200 family members (miR-200b, miR-200c, and miR-429), and two miR-30 family members (miR-30a-3p and miR-30e-3p). Lu et al. (2016) found that miR-505 regulates proliferation, invasion, and EMT in MHCC97 hepatoma cells by targeting high-mobility group box 1 (HMGB1). Ding et al. (2012) showed that the combination of a DNA methyltransferase (DNMT) inhibitor and upregulation of miR-200b could block lung metastasis of mesenchymal-phenotype hepatocellular carcinoma. Wang et al. (2014) indicated that miR-30a-3p inhibits tumor proliferation, invasion, and migration, and is downregulated in LIHC. Our data indicated that V\$FREAC4_01, V\$HOX13_01, and V\$SOX9_B1 may be key transcription factors in the regulation of PDZD11. Liu et al. (2016) demonstrated that Sox9 regulates self-renewal and tumorigenicity by promoting symmetrical cell division of cancer stem cells in LIHC. Taken together, abnormal expression of PDZD11 may modulate tumor cell proliferation, invasion, metastasis, and the development of LIHC by regulating these targets. Further studies are required to verify this hypothesis.

The emergence and development of LIHC are accompanied by a persistent inflammatory reaction. Inflammatory cells in the tumor microenvironment of LIHC mainly include macrophages, infiltrating lymphocytes, neutrophils, mast cells, dendritic cells, and eosinophils (Kim and Bae, 2016; Yan et al., 2018). In particular, Liu W.R. et al. (2020) reported that among these tumor-related regulatory $\mathrm{T}$ cells (Tregs), macrophages, and neutrophils are strongly correlated with OS and relapse-free survival (RFS) in LIHC patients. Here, we found that PDZD11 expression in LIHC was positively correlated with infiltrating levels of six immune cell types (i.e., B cells, $\mathrm{CD} 4^{+} \mathrm{T}$ cells,
CD8 + T cells, macrophages, neutrophils, and dendritic cells). Moreover, under the premise of high expression of PDZD11 mRNA in LIHC, the higher infiltration levels of the CD4 ${ }^{+}$ memory resting $\mathrm{T}$ cell subset were favorable factors for prognosis in LIHC patients. In contrast, the higher infiltration levels of the macrophage M2 subset had an unfavorable prognosis in LIHC (Figure 7). Previous studies have shown that $\mathrm{CD} 4^{+} \mathrm{T}$ cells and tumor-associated macrophages (TAMs) play a central role in pro-tumor immunity; their interactions with tumor cells can directly promote tumor growth, progression, invasion, and metastasis. Conversely, $\mathrm{CD}^{+} \mathrm{T}$ cells are responsible for antitumor responses, and increased $\mathrm{CD}^{+} \mathrm{T}$ cell infiltration usually indicates a better prognosis in LIHC (Yan et al., 2018; Ansari et al., 2020; Li et al., 2020). In summary, these data indicate that PDZD11 is not only a prognostic biomarker, but may also reflect the immune status of LIHC patients.

In summary, these findings highlight the critical role of PDZD11 in the development and progression of LIHC. However, immunohistochemistry and functional analysis are needed in future studies to verify the relationship between PDZD11 and EMT in LIHC at the clinical and cellular levels. In particular, compared with normal human hepatocytes, the overexpression level of PDZD11 mRNA was significantly higher than its protein level. In addition, the protein expression level of PDZD11 in HepG2 cells was significantly reduced. Therefore, overexpression of PDZD11 in LIHC could not be ruled out, which is a selfprotective feedback regulation mechanism that inhibits tumor metastasis. Further studies are needed to determine whether the aberrant expression of PDZD11 is detrimental or beneficial to patients with LIHC, and further studies are needed to explore how the aberrant expression of PDZD11 regulates the onset and progression of LIHC via EMT and OXPHOS pathways.

\section{DATA AVAILABILITY STATEMENT}

The original contributions presented in the study are included in the article/Supplementary Material, further inquiries can be directed to the corresponding author/s.

\section{ETHICS STATEMENT}

The studies involving human participants were reviewed and approved by the board of directors and the ethics committee of the First Affiliated Hospital of Wenzhou Medical University. The patients/participants provided their written informed consent to participate in this study.

\section{AUTHOR CONTRIBUTIONS}

SY and YP designed and supervised the study. YC and XY collected the patient samples and performed the study. YC, HX, TX, YP, and SY participated in data analysis and figure preparation. YP, SY, and TX revised the article was written. 
SY and YP reviewed the manuscript. All authors read and approved the final manuscript.

\section{FUNDING}

This study was supported by the Zhejiang Medical and Health Science and Technology Plan Project, No. 2016KYB191 and Key Discipline of Zhejiang Province in Medical Technology (first class, category A).

\section{SUPPLEMENTARY MATERIAL}

The Supplementary Material for this article can be found online at: https://www.frontiersin.org/articles/10.3389/fgene. 2021.669928/full\#supplementary-material

Supplementary Figure 1 | Protein-protein interaction (PPI) network of CDK5 Kinase-target networks (STRING).

\section{REFERENCES}

Ansari, R. E., Craze, M. L., Althobiti, M., Alfarsi, L., Ellis, I. O., Rakha, E. A. et al. (2020). Enhanced glutamine uptake influences composition of immune cell infiltrates in breast cancer. Br. J. Cancer 122, 94-101. doi: 10.1038/s41416019-0626-z

Bray, F., Ferlay, J., Soerjomataram, I., Siegel, R. L., Torre, L. A., and Jemal, A. (2018). Global cancer statistics 2018: GLOBOCAN estimates of incidence and mortality worldwide for 36 cancers in 185 countries. CA Cancer J. Clin. 68, 394-424. doi: 10.3322/caac. 21492

Cano, A., Pérez-Moreno, M. A., Rodrigo, I., Locascio, A., Blanco, M. J., del Barrio, M. G., et al. (2000). The transcription factor snail controls epithelialmesenchymal transitions by repressing E-cadherin expression. Nat. Cell Biol. 2, 76-83. doi: 10.1038/35000025

Chaffer, C. L., and Weinberg, R. A. (2011). A perspective on cancer cell metastasis. Science 331, 1559-1564. doi: 10.1126/science.1203543

Chandrashekar, D. S., Bashel, B., Balasubramanya, S. A. H., Creighton, C. J., PonceRodriguez, I., Chakravarthi, B., et al. (2017). UALCAN: a portal for facilitating tumor subgroup gene expression and survival analyses. Neoplasia 19, 649-658. doi: 10.1016/j.neo.2017.05.002

Ding, W., Dang, H., You, H., Steinway, S., Takahashi, Y., Wang, H. G., et al. (2012). miR-200b restoration and DNA methyltransferase inhibitor block lung metastasis of mesenchymal-phenotype hepatocellular carcinoma. Oncogenesis 1:e15. doi: 10.1038/oncsis.2012.15

Dongre, A., and Weinberg, R. A. (2019). New insights into the mechanisms of epithelial-mesenchymal transition and implications for cancer. Nat. Rev. Mol. Cell Biol. 20, 69-84. doi: 10.1038/s41580-018-0080-4

Ehrlich, S. M., Liebl, J., Ardelt, M. A., Lehr, T., De Toni, E. N., Mayr, D., et al. (2015). Targeting cyclin dependent kinase 5 in hepatocellular carcinoma-a novel therapeutic approach. J. Hepatol. 63, 102-113. doi: 10.1016/j.jhep.2015. 01.031

European Association for the Study of the Liver (EASL), (2018). EASL clinical practice guidelines: management of hepatocellular carcinoma. J. Hepatol. 69, 182-236. doi: 10.1016/j.jhep.2018.03.019

Fernandez-Moreira, D., Ugalde, C., Smeets, R., Rodenburg, R. J., Lopez-Laso, E., Ruiz-Falco, M. L., et al. (2007). X-linked NDUFA1 gene mutations associated with mitochondrial encephalomyopathy. Ann. Neurol. 61, 73-83.

Gao, J., Aksoy, B. A., Dogrusoz, U., Dresdner, G., Gross, B., Sumer, S. O., et al. (2013). Integrative analysis of complex cancer genomics and clinical profiles using the cBioPortal. Sci. Signal. 6:2004088.

Giannelli, G., Koudelkova, P., Dituri, F., and Mikulits, W. (2016). Role of epithelial to mesenchymal transition in hepatocellular carcinoma. J. Hepatol. 65, 798-808.
Supplementary Figure 2 | PPI network of MIR-505 (STRING).

Supplementary Figure 3 | PPI network of V\$FREAC4_01 transcription factor -target networks (STRING).

Supplementary Table 1 | Significantly enriched GO annotations (biological processes) of PDZD11 in LIHC (LinkedOmics).

Supplementary Table 2 | Significantly enriched GO annotations (cellular components) of PDZD11 in LIHC (LinkedOmics).

Supplementary Table 3 | Significantly enriched GO annotations (molecular functions) of PDZD11 in LIHC (LinkedOmics).

Supplementary Table 4 | Significantly enriched KEGG pathway annotations of PDZD11 in LIHC (LinkedOmics).

Supplementary Table $\mathbf{5}$ | Significantly enriched kinase-target networks of PDZD11 in LIHC (LinkedOmics).

Supplementary Table 6 | Significantly enriched miRNA-target networks of PDZD11 in LIHC (LinkedOmics).

Supplementary Table 7 | Significantly enriched transcription factor-target networks of PDZD11 in LIHC (LinkedOmics).

Goellner, G. M., DeMarco, S. J., and Strehler, E. E. (2003). Characterization of PISP, a novel single-PDZ protein that binds to all plasma membrane Ca2+-ATPase b-splice variants. Ann. N. Y. Acad. Sci. 986, 461-471. doi: 10.1111/j.1749-6632. 2003.tb07230.x

Guerrera, D., Shah, J., Vasileva, E., Sluysmans, S., Méan, I., Jond, L., et al. (2016). PLEKHA7 Recruits PDZD11 to adherens junctions to stabilize nectins. J. Biol. Chem. 291, 11016-11029. doi: 10.1074/jbc.M115.712935

Huang da, W., Sherman, B. T., and Lempicki, R. A. (2009). Systematic and integrative analysis of large gene lists using DAVID bioinformatics resources. Nat. Protoc. 4, 44-57. doi: 10.1038/nprot.2008.211

Huang, I. T., Dhungel, B., Shrestha, R., Bridle, K. R., Crawford, D. H. G., Jayachandran, A., et al. (2019). Spotlight on bortezomib: potential in the treatment of hepatocellular carcinoma. Expert Opin. Investig. Drugs 28, 7-18. doi: 10.1080/13543784.2019.1551359

Jemal, A., Ward, E. M., Johnson, C. J., Cronin, K. A., Ma, J., Ryerson, B., et al. (2017). Annual report to the nation on the status of cancer, 1975-2014, featuring survival. J. Natl. Cancer Inst. 109:djx030.

Jiang, Y., Sun, A., Zhao, Y., Ying, W., Sun, H., Yang, X., et al. (2019). Proteomics identifies new therapeutic targets of early-stage hepatocellular carcinoma. Nature 567, 257-261.

Jung, K. H., Kim, J. K., Noh, J. H., Eun, J. W., Bae, H. J., Xie, H. J., et al. (2010). Targeted disruption of Nemo-like kinase inhibits tumor cell growth by simultaneous suppression of cyclin D1 and CDK2 in human hepatocellular carcinoma. J. Cell Biochem. 110, 687-696. doi: 10.1002/jcb.22579

Kim, J., and Bae, J. S. (2016). Tumor-associated macrophages and neutrophils in tumor microenvironment. Mediators Inflamm. 2016:6058147. doi: 10.1155/ 2016/6058147

Konopka, G., Tekiela, J., Iverson, M., Wells, C., and Duncan, S. A. (2007). Junctional adhesion molecule-A is critical for the formation of pseudocanaliculi and modulates E-cadherin expression in hepatic cells. J. Biol. Chem. 282, 28137-28148. doi: 10.1074/jbc.M703592200

Lara-Pezzi, E., Roche, S., Andrisani, O. M., Sánchez-Madrid, F., and LópezCabrera, M. (2001). The hepatitis B virus HBx protein induces adherens junction disruption in a src-dependent manner. Oncogene 20, 3323-3331. doi: 10.1038/sj.onc. 1204451

Lee, Y. R., Khan, K., Armfield-Uhas, K., Srikanth, S., Thompson, N. A., Pardo, M., et al. (2020). Mutations in FAM50A suggest that Armfield XLID syndrome is a spliceosomopathy. Nat. Commun. 11:3698. doi: 10.1038/s41467-02017452-6

Li, R., Liu, H., Cao, Y., Wang, J., Chen, Y., Qi, Y., et al. (2020). Identification and validation of an immunogenic subtype of gastric cancer with abundant intratumoural CD103(+)CD8(+) T cells conferring favourable prognosis. $\mathrm{Br}$. J. Cancer 122, 1525-1534. doi: 10.1038/s41416-020-0813-y 
Li, T., Fan, J., Wang, B., Traugh, N., Chen, Q., Liu, J. S., et al. (2017). TIMER: a web server for comprehensive analysis of tumor-infiltrating immune cells. Cancer Res. 77, e108-e110.

Liu, C., Liu, L., Chen, X., Cheng, J., Zhang, H., Shen, J., et al. (2016). Sox9 regulates self-renewal and tumorigenicity by promoting symmetrical cell division of cancer stem cells in hepatocellular carcinoma. Hepatology 64, 117-129. doi: 10.1002/hep. 28509

Liu, S. H., Shen, P. C., Chen, C. Y., Hsu, A. N., Cho, Y. C., Lai, Y. L., et al. (2020). DriverDBv3: a multi-omics database for cancer driver gene research. Nucleic Acids Res. 48, D863-D870.

Liu, W. R., Tian, M. X., Tang, Z., Fang, Y., Zhou, Y. F., Song, S. S., et al. (2020). Nine-factor-based immunohistochemistry classifier predicts recurrence for early-stage hepatocellular carcinoma after curative resection. Br. J. Cancer 123, 92-100. doi: 10.1038/s41416-020-0864-0

Lu, L., Qiu, C., Li, D., Bai, G., Liang, J., and Yang, Q. (2016). MicroRNA-505 suppresses proliferation and invasion in hepatoma cells by directly targeting high-mobility group box 1. Life Sci. 157, 12-18. doi: 10.1016/j.lfs.2016.05.039

Nabokina, S. M., Subramanian, V. S., and Said, H. M. (2011). Association of PDZcontaining protein PDZD11 with the human sodium-dependent multivitamin transporter. Am. J. Physiol. Gastrointest Liver Physiol. 300:23.

Okajima, W., Komatsu, S., Ichikawa, D., Miyamae, M., Ohashi, T., Imamura, T., et al. (2017). Liquid biopsy in patients with hepatocellular carcinoma: circulating tumor cells and cell-free nucleic acids. World J. Gastroenterol. 23, 5650-5668. doi: 10.3748/wjg.v23.i31.5650

Park, S. Y., Shin, J. H., and Kee, S. H. (2017). E-cadherin expression increases cell proliferation by regulating energy metabolism through nuclear factor- $\kappa \mathrm{B}$ in AGS cells. Cancer Sci. 108, 1769-1777. doi: 10.1111/cas.13321

Rhodes, D. R., Kalyana-Sundaram, S., Mahavisno, V., Varambally, R., Yu, J., Briggs, B. B., et al. (2007). Oncomine 3.0: genes, pathways, and networks in a collection of 18,000 cancer gene expression profiles. Neoplasia 9, 166-180. doi: 10.1593/ neo. 07112

Rouaud, F., Tessaro, F., Aimaretti, L., Scapozza, L., and Citi, S. (2020). Cooperative binding of the tandem WW domains of PLEKHA7 to PDZD11 promotes conformation-dependent interaction with tetraspanin 33. J. Biol. Chem. 295, 9299-9312. doi: 10.1074/jbc.RA120.012987

Schmidt, K., Ralle, M., Schaffer, T., Jayakanthan, S., Bari, B., Muchenditsi, A., et al. (2018). ATP7A and ATP7B copper transporters have distinct functions in the regulation of neuronal dopamine- $\beta$-hydroxylase. J. Biol. Chem. 293, 20085-20098. doi: 10.1074/jbc.ra118.004889

Shah, J., Guerrera, D., Vasileva, E., Sluysmans, S., Bertels, E., and Citi, S. (2016). PLEKHA7: cytoskeletal adaptor protein at center stage in junctional organization and signaling. Int. J. Biochem. Cell Biol. 75, 112-116. doi: 10.1016/ j.biocel.2016.04.001

Siegel, R. L., Miller, K. D., and Jemal, A. (2019). Cancer statistics, 2019. CA Cancer J. Clin. 69, 7-34.

Stephenson, S. E., Dubach, D., Lim, C. M., Mercer, J. F., and La Fontaine, S. (2005). A single PDZ domain protein interacts with the Menkes copper ATPase, ATP7A. a new protein implicated in copper homeostasis. J. Biol. Chem. 280, 33270-33279. doi: 10.1074/jbc.m505889200
Tang, Z., Li, C., Kang, B., Gao, G., and Zhang, Z. (2017). GEPIA: a web server for cancer and normal gene expression profiling and interactive analyses. Nucleic Acids Res. 45, W98-W102.

Thiery, J. P., Acloque, H., Huang, R. Y., and Nieto, M. A. (2009). Epithelialmesenchymal transitions in development and disease. Cell 139, 871-890. doi: 10.1016/j.cell.2009.11.007

Vasaikar, S. V., Straub, P., Wang, J., and Zhang, B. (2018). LinkedOmics: analyzing multi-omics data within and across 32 cancer types. Nucleic Acids Res. 46, D956-D963.

Vasileva, E., Sluysmans, S., Bochaton-Piallat, M. L., and Citi, S. (2017). Cell-specific diversity in the expression and organization of cytoplasmic plaque proteins of apical junctions. Ann. N. Y. Acad. Sci. 1405, 160-176. doi: 10.1111/nyas.13391

Villanueva, A. (2019). Hepatocellular carcinoma. N. Engl. J. Med. 380, 1450-1462. von Mering, C., Huynen, M., Jaeggi, D., Schmidt, S., Bork, P., and Snel, B. (2003). STRING: a database of predicted functional associations between proteins. Nucleic Acids Res. 31, 258-261. doi: 10.1093/nar/gkg034

Wan, L. C., Maisonneuve, P., Szilard, R. K., Lambert, J. P., Ng, T. F., Manczyk, N., et al. (2017). Proteomic analysis of the human KEOPS complex identifies C14ORF142 as a core subunit homologous to yeast Gon7. Nucleic Acids Res. 45, 805-817. doi: 10.1093/nar/gkw1181

Wang, W., Lin, H., Zhou, L., Zhu, Q., Gao, S., Xie, H., et al. (2014). MicroRNA$30 \mathrm{a}-3 \mathrm{p}$ inhibits tumor proliferation, invasiveness and metastasis and is downregulated in hepatocellular carcinoma. Eur. J. Surg. Oncol. 40, 1586-1594. doi: 10.1016/j.ejso.2013.11.008

Warde-Farley, D., Donaldson, S. L., Comes, O., Zuberi, K., Badrawi, R., Chao, P., et al. (2010). The GeneMANIA prediction server: biological network integration for gene prioritization and predicting gene function. Nucleic Acids Res. 38, W214-W220.

Yamada, S., Okumura, N., Wei, L., Fuchs, B. C., Fujii, T., Sugimoto, H., et al. (2014). Epithelial to mesenchymal transition is associated with shorter diseasefree survival in hepatocellular carcinoma. Ann. Surg. Oncol. 21, 3882-3890. doi: 10.1245/s10434-014-3779-2

Yan, L., Xu, F., and Dai, C. L. (2018). Relationship between epithelialto-mesenchymal transition and the inflammatory microenvironment of hepatocellular carcinoma. J. Exp. Clin. Cancer Res. 37:203.

Yang, Z., Liu, S., Zhu, M., Zhang, H., Wang, J., Xu, Q., et al. (2016). PS341 inhibits hepatocellular and colorectal cancer cells through the FOXO3/CTNNB1 signaling pathway. Sci. Rep. 6:22090. doi: 10.1038/srep22090

Conflict of Interest: The authors declare that the research was conducted in the absence of any commercial or financial relationships that could be construed as a potential conflict of interest.

Copyright (c) 2021 Chen, Xie, Xie, Yang, Pang and Ye. This is an open-access article distributed under the terms of the Creative Commons Attribution License (CC BY). The use, distribution or reproduction in other forums is permitted, provided the original author(s) and the copyright owner(s) are credited and that the original publication in this journal is cited, in accordance with accepted academic practice. No use, distribution or reproduction is permitted which does not comply with these terms. 